\title{
Danthron activates AMP-activated protein kinase and regulates lipid and glucose metabolism in vitro
}

\author{
Rong ZHOU ${ }^{1}$, Ling WANG ${ }^{1}$, Xing XU² ${ }^{2}$ Jing $\mathrm{CHEN}^{2}$, Li-hong $\mathrm{HU}^{2}$, Li-li CHEN ${ }^{2, *}$, Xu SHEN ${ }^{1,2, *}$ \\ ${ }^{1}$ School of Pharmacy, East China University of Science and Technology, Shanghai 200237, China; ${ }^{2}$ State Key Laboratory of Drug \\ Research, Shanghai Institute of Materia Medica, Chinese Academy of Sciences, Shanghai 201203, China
}

\begin{abstract}
Aim: To discover the active compound on AMP-activated protein kinase (AMPK) activation and investigate the effects of the active compound 1,8-dihydroxyanthraquinone (danthron) from the traditional Chinese medicine rhubarb on AMPK-mediated lipid and glucose metabolism in vitro.

Methods: HepG2 and C2C12 cells were used. Cell viability was determined using MTT assay. Real-time PCR was performed to measure the gene expression. Western blotting assay was applied to investigate the protein phosphorylation level. Enzymatic assay kits were used to detect the total cholesterol (TC), triglyceride (TG) and glucose contents.

Results: Danthron $(0.1,1$, and $10 \mu \mathrm{mol} / \mathrm{L})$ dose-dependently promoted the phosphorylation of AMPK and acetyl-CoA carboxylase (ACC) in both HepG2 and C2C12 cells. Meanwhile, danthron treatment significantly reduced the lipid synthesis related sterol regulatory element-binding protein 1c (SREBP1c) and fatty acid synthetase (FAS) gene expressions, and the TC and TG levels. In addition, danthron treatment efficiently increased glucose consumption. The actions of danthron on lipid and glucose metabolism were abolished or reversed by co-treatment with the AMPK inhibitor compound C.

Conclusion: Danthron effectively reduces intracellular lipid contents and enhanced glucose consumption in vitro via activation of AMPK signaling pathway.
\end{abstract}

Keywords: 1,8-dihydroxyanthraquinone (danthron); traditional Chinese medicine; AMPK; SREBP1c; fatty acid synthetase; cholesterol; triglyceride; glucose consumption

Acta Pharmacologica Sinica (2013) 34: 1061-1069; doi: 10.1038/aps.2013.39; published online 17 Jun 2013

\section{Introduction}

Impaired glucose and lipid metabolism occur commonly in several major diseases such as obesity, type 2 diabetes, hypertension and atherogenic dyslipidemia ${ }^{[1,2]}$. AMP-activated protein kinase (AMPK), which is expressed in the liver, brain, and skeletal muscle tissues, has been a key sensor in the regulation of fatty acid and glucose homeostasis ${ }^{[3]}$ and a potential therapeutic target for the prevention and treatment of diabetes ${ }^{[4,5]}$. AMPK becomes activated when phosphorylation takes place at threonine-172 by the AMPK kinases ${ }^{[6]}$. In the liver, AMPK activation increases fatty acid oxidation and inhibits cholesterol synthesis, lipogenesis, triglyceride synthesis and gluconeogenesis. In muscle, AMPK promotes fatty acid oxidation and glucose uptake. In adipose tissue, activated AMPK increases fatty acid oxidation and glucose uptake while atten-

\footnotetext{
* To whom correspondence should be addressed.

E-mail lilichen@simm.ac.cn (Li-li CHEN); xshen@simm.ac.cn (Xu SHEN)

Received 2013-01-11 Accepted 2013-03-20
}

uating adipocyte lipolysis and lipogenesis ${ }^{[3-5]}$.

Acetyl-Coenzyme A carboxylase (ACC) is a direct downstream target of AMPK, and its active dephosphorylated form can catalyze the conversion of acetyl-CoA to malonyl-CoA in the de novo lipid synthesis pathway. AMPK phosphorylates and inactivates ACC, which inhibits the conversion from acetyl-CoA to malonyl-CoA, and thus increases the transportation of fatty acids into the mitochondria for oxidation ${ }^{[7]}$. Meanwhile, AMPK activation reduces the expression of sterol regulatory element-binding protein $1 \mathrm{c}$ (SREBP1c) and its downstream gene fatty acid synthetase (FAS) to control triglyceride synthesis ${ }^{[4]}$. Moreover, activated AMPK regulates cholesterol synthesis by phosphorylating and inactivating 3-hydroxy-3-methylglutaryl-CoA reductase ${ }^{[4]}$. In addition, in adipose and skeletal muscle tissues, AMPK promotes the translocation of glucose transporter type 4 (Glut4) to the cellular membrane to transport glucose ${ }^{[8]}$. In the liver, AMPK helps regulate glucose homeostasis by reducing the gluconeogenic key genes including phosphoenolpyruvate carboxykinase (PEPCK) and glucose 6-phosphatase (G6Pase) ${ }^{[9,10]}$. Further- 
more, the AMPK activator AICAR ${ }^{[11]}$ could enhance the Glut2dependent glucose uptake in the jejunum, implying a possible new role for AMPK ${ }^{[12]}$.

Due to the important regulation of AMPK on glucose and lipid metabolism, novel agents to activate AMPK were of great interest. Currently, in addition to known activators such as $\operatorname{AICAR}^{[11]}$, metformin (Met $)^{[13]}$, berberine ${ }^{[14]}$, and A-769662 $2^{[10]}$, more active natural products have been increasingly discovered from the plant kingdom. In particular, many effective AMPK activators have come from traditional herbs ${ }^{[15-17]}$, such as the natural anthraquinone derivative emodin, which ameliorates dyslipidemia in high-fat-diet rats by activating $\mathrm{AMPK}^{[15]}$. As with emodin, 1,8-dihydroxyanthraquinone (danthron, Figure 1A) is also a natural anthraquinone derivative extracted from the traditional Chinese medicine rhubarb. Given the structural similarity of these anthraquinones, we thus explored the potential role of danthron in regulating AMPK activation.

Previously, danthron was used as a laxative $\mathrm{e}^{[18]}$. Since danthron induced DNA damage and inhibited DNA repair, it exhibited efficient anticancer ${ }^{[19]}$, antiproliferative ${ }^{[20]}$ and antimetastatic ${ }^{[20]}$ effects. In addition, we previously found that danthron was a retinoic $X$ receptor antagonist and that it helped improve insulin resistance in high-fat diet-induced obesity mice ${ }^{[21]}$. Our current work shows that danthron functions in regulating glucose and lipid metabolism by activating AMPK in both HepG2 and C2C12 cells. These findings prolong the pharmacological actions of danthron and provide a new clue for anti-diabetic drug development.

\section{Methods and materials}

\section{Reagents and plasmids}

Cell culture plastic ware was purchased from Corning Inc (Corning, New York, USA). All cell culture media and sera were purchased from Invitrogen (Carlsbad, CA, USA). Danthron and guggulsterone (GS) were purchased from J\&J\&K Chemical Ltd (Beijing, China); Metformin (Met), compound C, TO901317 (TO90) and methylthiazolyldiphenyl-tetrazolium bromide (MTT) were obtained from Sigma-Aldrich (St Louis, MI, USA); RNAiso reagent kit, PrimeScriptTMRT reagent kit, and SYBR Premix Ex Taq Real-time PCR master mix were from TakaRa (Otsu, Shiga, Japan). Anti-GAPDH antibody was from KangCheng Bio-tech (Shanghai, China). The other antibodies were from Cell Signaling Technology (Beverly, Massachusetts, USA). The SuperSignal West Dura and Pico substrates were from Thermo Fisher (San Jose, CA, USA). Lipofectamine 2000 Transfection Reagent was from Invitrogen. The Dual Luciferase Reporter Assay kit was from Promega (Madison, Wisconsin, USA). Other solvents and reagents were purchased in analytical grade and used without further purifications. The pGL3-ABCA1-promoter-luc, pGL3-ABCG1promoter-luc and pRL-SV40 plasmids were kindly donated by Prof Gerd SCHMITZ (Institute for Clinical Chemistry, University of Regensburg), Prof Steven L SABOL (Laboratory of Developmental Biology NHLBI Division of Intramural Research) and Prof J Larry JAMESON (Department of Medi- cine, Northwestern Memorial Hospital), respectively.

\section{Cell culture and differentiation}

HepG2 cells were cultured in MEM medium, and C2C12 cells were cultured in DMEM medium. All cells were cultured at $37^{\circ} \mathrm{C}$ in media supplemented with $10 \%$ FBS and in a humidified atmosphere with $5 \% \mathrm{CO}_{2}$. After $100 \%$ confluence, $\mathrm{C} 2 \mathrm{C} 12$ cells were differentiated using DMEM containing $2 \%$ horse serum for $4 \mathrm{~d}$ to obtain mature myotubes.

\section{Cell viability assay}

Cells were treated with the indicated compounds for 24 or $48 \mathrm{~h}$, and then incubated with $0.5 \mathrm{mg} / \mathrm{mL}$ MTT in culture media for another $4 \mathrm{~h}$. Cell viability was evaluated using MTT assay as previously described ${ }^{[22]}$.

\section{Transient transfection and promoter activity measurement}

HepG2 cells were transfected with pGL3-ABCA1 promoterluc or pGL3-ABCG1 promoter-luc and pRL-SV40 plasmids using Lipofectamine 2000 Transfection Reagent according to the manufacturer's protocols. At $6 \mathrm{~h}$ post-transfection, the cells were incubated with danthron $(0-20 \mu \mathrm{mol} / \mathrm{L})$, TO90 (2 $\mu \mathrm{mol} / \mathrm{L}$ ) or DMSO for $24 \mathrm{~h}$. Luciferase activity was measured using the Dual Luciferase Reporter Assay kit.

\section{Western blotting}

Cells were harvested and separated by SDS-PAGE, and then transferred into the nitrocellulose membrane. Corresponding antibodies were incubated, and the SuperSignal West Dura (and Pico) substrates were applied for signal detection. ImageQuant LAS 4000 mini (GE Health) was used for signal collection.

\section{Real-time RT-PCR}

Starved overnight, cells were incubated with the compounds for $24 \mathrm{~h}$, and then total RNA was extracted and reversed using a corresponding reagent kit (TAKARA). Real-time PCR was performed using SYBR Green Real-time PCR master mix on the DNA Engine Opticon 2 System (Bio-Rad Laboratories, USA). The relative mRNA levels were normalized to $\beta$-actin or GAPDH. PCR primer pairs are listed in Table 1.

\section{$\mathrm{TG} / \mathrm{TC}$ content measurement}

HepG2 and mature C2C12 cells were starved overnight and treated with indicated compounds in a FBS-free medium for $24 \mathrm{~h}$. The samples' harvest, treatment and detection were performed as described previously ${ }^{[23]}$. Cellular total cholesterol (TC) and triglycerides (TG) were measured using the kits (Beihua-kangtai Clinical Reagent Limited Company, Beijing, China).

\section{Glucose consumption assay}

HepG2 and mature C2C12 cells were planted in 96-well plates with 6 blank wells without cells as the control. After the cells reached $80 \%$ confluence, the medium was replaced by phenol red- and FBS-free DMEM adding $11.1 \mathrm{mmol} / \mathrm{L}$ glucose and 
Table 1. The primer pairs sequences.

\begin{tabular}{lll}
\hline Gene & \multicolumn{1}{c}{ Forward primer $\left(5^{\prime}-3^{\prime}\right)$} & \multicolumn{1}{c}{ Reverse primer $\left(5^{\prime}-3^{\prime}\right)$} \\
\hline SREBP1C $^{\text {a }}$ & GGATTGCACTTTCGAAGACATG & AGGATGCTCAGTGGCACTG \\
FAS $^{\text {a }}$ & TGTGGACATGGTCACGGAC & GGCATCAACCTAGACAGGTC \\
ABCA1 $^{\text {a }}$ & ACCCACCCTATGAACAACATGA & GAGTCGGGTAACGGAACAGG \\
ABCG1 $^{\text {a }}$ & CGTGCGCTTTGGCTGTTT & CCACTGTAGGTACGTGGGGAT \\
B-actin $^{\text {a }}$ & CATGTACGTTGCTATCCAGGC & CTCCTTAATGACGCACGAT \\
SREBP1C $^{\text {b }}$ & CCGGGAGGCTTCTCTACAG & GGTCACAGTGGTCGTTACAGG \\
FAS $^{\text {b }}$ & GGCTCTATGGATTACCCAAGC & CCAGTGTTCGTTCCTCGGA \\
ABCA1 $^{\text {b }}$ & GCTTGTTGGCCTCAGTTAAGG & GTAGCTCAGGCGTACAGAGAT \\
ABCG1 $^{\text {b }}$ & CTTTCCTACTCTGTACCCGAGG & CGGGGCATTCCATTGATAAGG \\
GAPDH $^{\text {b }}$ & ACAGCAACAGGGTGGTGGAC & TTTGAGGGTGCAGCGAACTT \\
\hline
\end{tabular}

${ }^{\mathrm{a}}$ homo sapiens primers; ${ }^{\mathrm{b}}$ mus musculus primers.

$0.2 \%$ BSA. Meanwhile, cells were incubated with the indicated compounds for $24 \mathrm{~h}$. The glucose content in the medium was then determined by the glucose oxidase method according to the manufacturer's protocol (Rsbio, Shanghai, China). Next, the MTT assay was performed to evaluate cell viability and adjust the glucose consumption values. Glucose consumption =glucose concentration of blank wells - glucose concentration of wells plated with cells.

\section{Statistical analysis}

Results were presented as mean $\pm S E M$ for at least three separate experiments in each group. Differences between groups were examined for statistical significance using Student's $t$-test or one-way ANOVA. The value of $P<0.05$ was considered to be a statistically significant difference.

\section{Results}

Danthron activated AMPK and attenuated lipid accumulation in HepG2 cells

To evaluate the effect of danthron on AMPK phosphorylation in HepG2 cells, phosphorylated AMPK (p-AMPK) and total AMPK (t-AMPK) were detected by Western blot assay. First, we identified the effect of danthron on HepG2 cell viability to determine the appropriate concentration for later experiments. We found that $10 \mu \mathrm{mol} / \mathrm{L}$ danthron/24 h might be safe for HepG2 cells (Figure 1C). With 80\% confluence, HepG2 cells were incubated with danthron (0.1-10 $\mu \mathrm{mol} / \mathrm{L})$ in FBS-Free media for $8 \mathrm{~h}$. Subsequently, cells were harvested for Western blot assay. As shown in Figure 1B, danthron increased the p-AMPK protein in a dose-dependent manner, and no changes in $\mathrm{t}$-AMPK protein were observed. It was suggested that danthron activated AMPK in HepG2 cells. In addition, we also detected the phosphorylation of ACC, which was the downstream protein regulated by AMPK. As expected, the phosphorylated ACC, but not the total ACC, was also augmented under the treatment of danthron (Figure 1B).

Given that AMPK modulated lipid biosynthesis by controlling its downstream gene expression at the transcriptional level, such as SREBP1c and FAS, we therefore analyzed the
mRNA levels of these genes. HepG2 cells were starved overnight and treated with different concentrations of danthron for another $24 \mathrm{~h}$. Both SREBP1c and FAS mRNA levels were attenuated by danthron in a dose-dependent manner (Figure 1D and 1E). Meanwhile, cells were treated as described above, and TG and TC contents were measured using GS as a positive control. As expected, $40 \mu \mathrm{mol} / \mathrm{L}$ GS obviously lowered both TG and TC contents. Similar actions were observed in danthron-treated groups in a dose-dependent manner (Figure $1 F$ and $1 G)$.

In addition, considering the protein expression of ABCA1 and ABCG1 regulated by AMPK in endothelial cells, we further explored the corresponding effects of danthron by determining their promoter activities in HepG2 cells. As shown in Figure $2 \mathrm{~A}$ and $2 \mathrm{~B}$, danthron increased the promoter activities of ABCA1 and ABCG1 dose-dependently. Meanwhile, we detected the effects of danthron on ABCA1 and ABCG1 endogenous expressions in HepG2 cells by RT-PCR assay. We found that the mRNA levels of the two genes were both increased with danthron treatment (Figure 2C and 2D).

All of the above-mentioned results suggested that danthron activated AMPK and regulated lipid accumulation in HepG2 cells.

\section{Danthron increased AMPK activation and attenuated lipid accumulation in $\mathrm{C} 2 \mathrm{C} 12$ cells}

Skeletal muscle is another important tissue that responds to the activation of AMPK as well as the liver. Thus, we detected the probable effects of danthron in C2C12 cells. Mature C2C12 cells were treated with FBS-free DMEM containing danthron (0.1-10 $\mu \mathrm{mol} / \mathrm{L})$ for $8 \mathrm{~h}$, and then Western blot assay was performed to analyze the changes in protein expression. As shown in Figure 3A, both AMPK and ACC phosphorylation levels were increased in a dose-dependent manner with the administration of danthron. In addition, SREBP1c and FAS mRNA levels were also determined. We found that danthron efficiently attenuated the gene expressions of SREBP1c and FAS in C2C12 cells (Figure 3B and 3C). Correspondingly, we observed the ABCA1 and ABCG1 mRNA levels, which were found to be modestly increased in danthron-treated cells (Figure 2E and 2F). Subsequently, we also determined the contents of intracellular TG and TC and observed their corresponding decreases in response to different concentrations of danthron (Figure 3D and 3E). These results suggested that danthron increased the activation of AMPK and further regulated lipid metabolism in $\mathrm{C} 2 \mathrm{C} 12$ cells.

\section{The regulation of danthron on lipid metabolism was AMPK- dependent}

To investigate whether the regulation of danthron was AMPKdependent, we next tested the corresponding effects of danthron on lipid metabolism by co-incubation with an AMPK inhibitor compound C. HepG2 cells were treated with FBSfree media containing the indicated compounds with or without compound $\mathrm{C}$ for $8 \mathrm{~h}$. The AMPK activator metformin (Met) was used as a positive control. We found that both dan- 
A<smiles>O=C1c2ccccc2C(=O)c2c(O)cccc21</smiles>

B

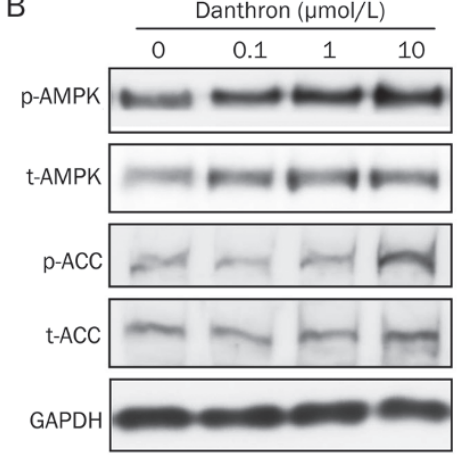

C

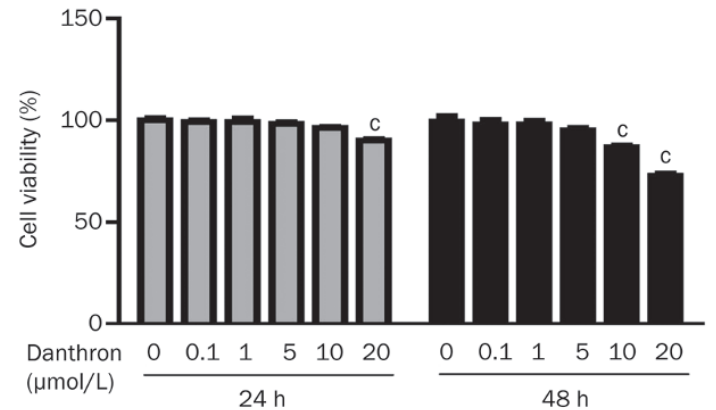

D

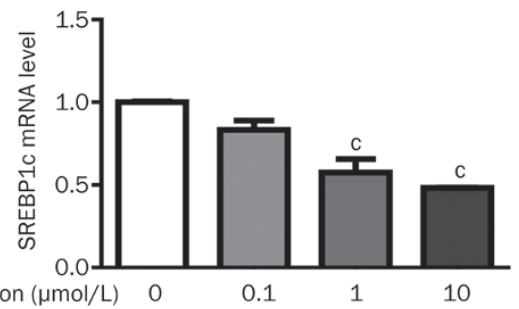

F

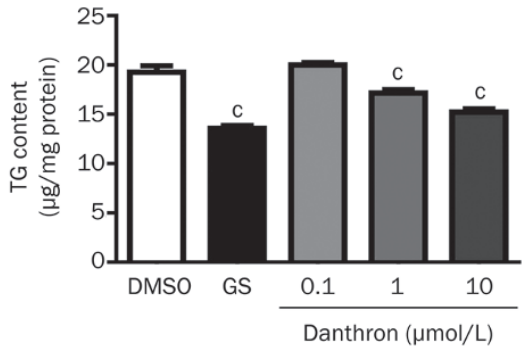

E

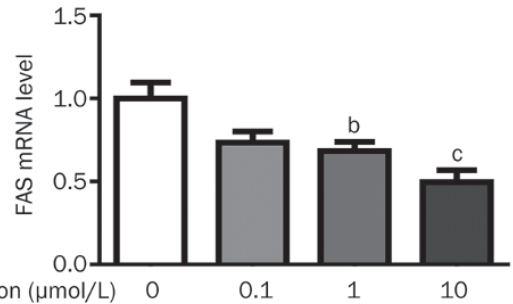

G

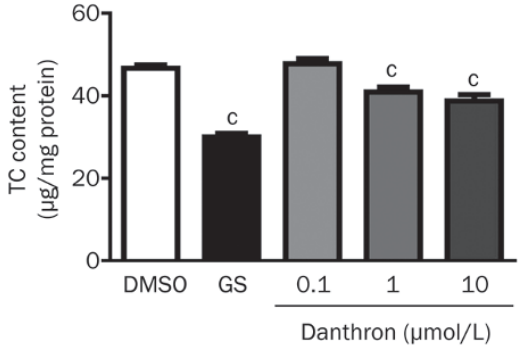

Figure 1. Regulation of danthron on AMPK activation and lipid accumulation in HepG2 cells. (A) Chemical structure of danthron. (B) Concentrationdependent effects of danthron on AMPK and ACC phosphorylation detected by Western blotting. (C) Effect of danthron on cell viability examined using the MTT assay. ( $D$ and $E$ ) The mRNA levels of SREBP1c and FAS determined by RT-PCR. ( $F$ and $G$ ) The levels of intracellular TG and TC measured by the enzymatic colorimetry method. GS $(40 \mu \mathrm{mol} / \mathrm{L})$ was used as a positive control. Data are presented as the mean $\pm S E M$ of three independent experiments. ${ }^{\mathrm{b}} P<0.05,{ }^{\mathrm{c}} P<0.01$ vs DMSO.

thron and Met could obviously enhance the phosphorylation of AMPK and ACC, and such increases were reversed totally or partially by the compound $\mathrm{C}$ treatment (Figure 4A). The effects of Met (Figure 4A) were in accordance with the previous results, thus indicating the effectiveness of our study. The SREBP1c and FAS gene expressions were also detected by the RT-PCR method. As shown in Figure $4 \mathrm{~B}$ and $4 \mathrm{C}$, both danthron and Met significantly reduced the mRNA levels of these genes, and the effects were more or less reversed by compound C.

Subsequently, we also determined the corresponding effects of danthron in C2C12 cells. Cell treatment and compounds administration were described above. As expected, we found increases in p-AMPK and p-ACC in danthron-treated cells and decreases in these proteins in compound C-treated cells (Figure 4D). Moreover, the danthron-induced increases in p-AMPK and p-ACC were obviously reversed after co-incu- bation with compound C (Figure 4D). Both danthron and Met reduced the mRNA levels of SREBP1c and FAS in C2C12 cells. Meanwhile, compound $\mathrm{C}$ reversed the effects of danthron on these gene expressions (Figure $4 \mathrm{E}$ and $4 \mathrm{~F}$ ).

These results suggest that danthron regulated the lipid metabolism-related genes primarily through the activation of AMPK.

\section{Danthron promoted glucose consumption in both HepG2 and} C2C12 cells

Because AMPK activation could modulate glucose metabolism, we investigated the potential role of danthron in glucose consumption in HepG2 and C2C12 cells. Cells were treated and measured as described in the Methods section. The effects of danthron and Met without or with compound $C$ are shown in Figure 5A and 5B (HepG2 cells) and Figure 5C and 5D (C2C12 cells). In both cell lines, as with Met, danthron 

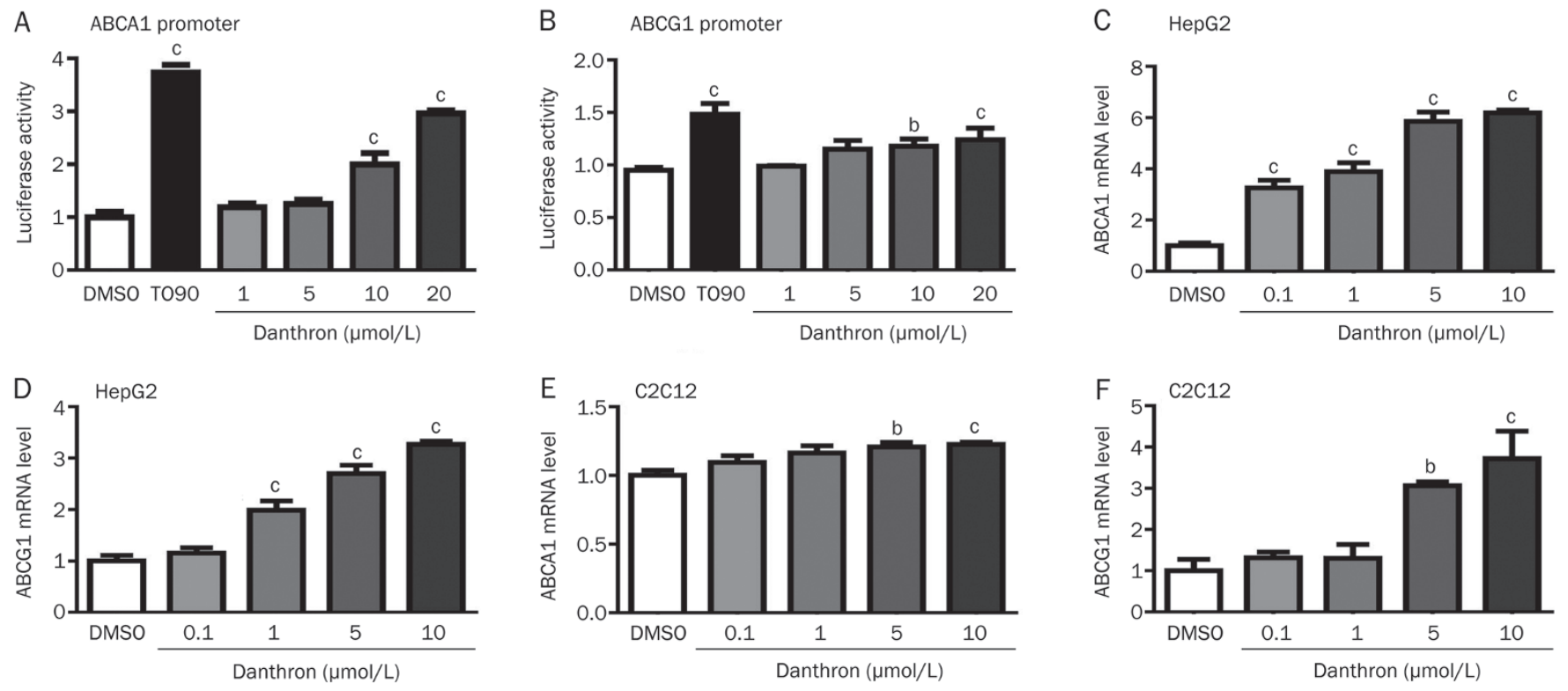

Figure 2. Increases in $A B C A 1$ and $A B C G 1$ promoter activities and gene expressions by danthron treatment. (A and B) The effects of danthron on $A B C A 1$ and ABCG1 promoter activities were determined by luciferase reporter gene-based assay. TO90 (T0901317, $2 \mu$ mol/L) was used as a positive control. $(C$ and $D)$ The gene expressions of $A B C A 1$ and $A B C G 1$ were detected in HepG2 cells. ( $E$ and $F$ ) The mRNA levels of ABCA1 and ABCG1 were tested in C2C12 cells. Data are presented as the mean \pm SEM of three independent experiments. ${ }^{b} P<0.05,{ }^{C} P<0.01$ vs DMSO.
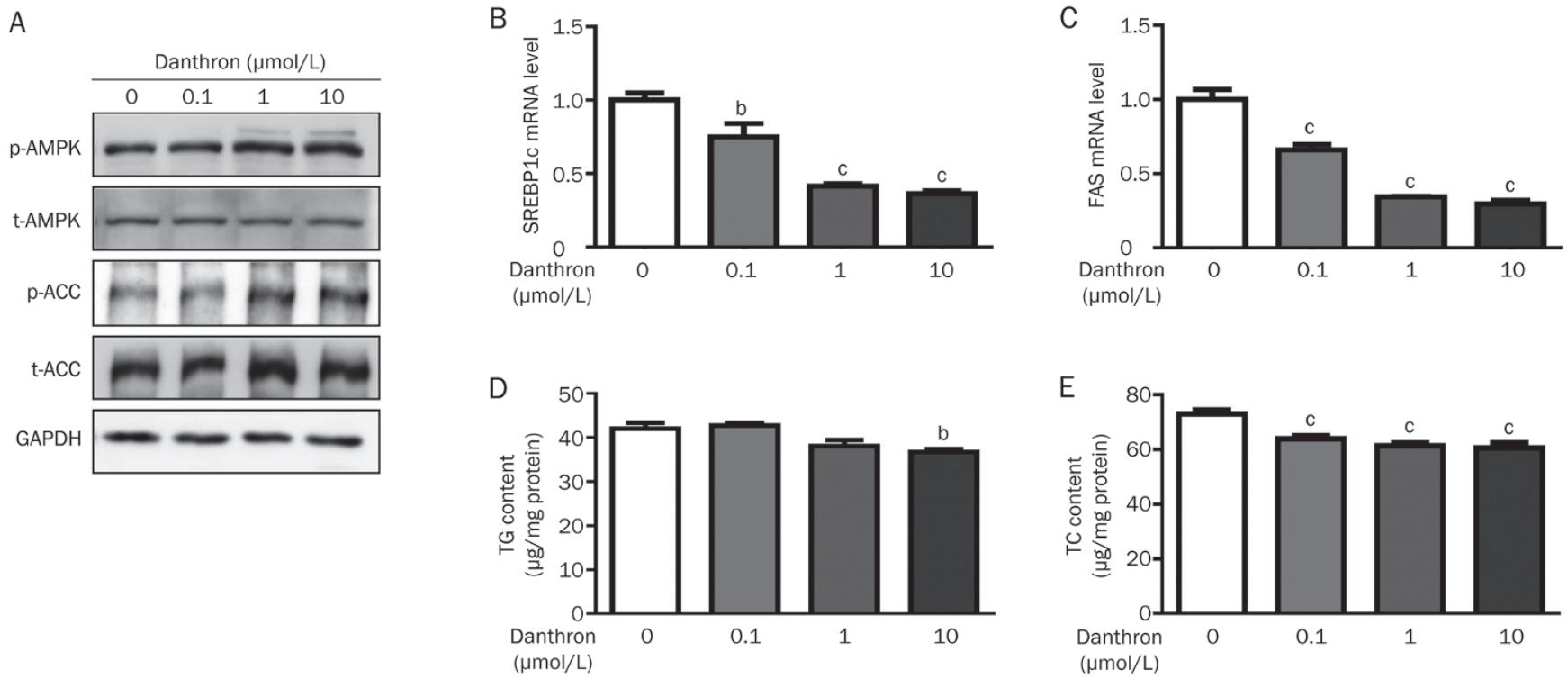

Figure 3. Effects of danthron on AMPK activation and lipid accumulation in mature C2C12 cells. (A) Danthron efficiently increased the phosphorylation of AMPK and ACC. (B and C) Concentration-dependent effects of danthron on SREBP1C and FAS gene expressions were determined. (D and E) Danthron attenuated the intracellular TG and TC contents. The concentrations of danthron were indicated. Data are presented as the mean \pm SEM of three independent experiments. ${ }^{\mathrm{b}} P<0.05,{ }^{\mathrm{c}} P<0.01$ vs DMSO.

increased glucose consumption in a dose-dependent manner (Figure 5A and 5C). Furthermore, glucose uptake was attenuated under the treatment of danthron or Met co-incubating with compound C (Figure 5B and 5D).

In addition, we also detected the effects of danthron on the key proteins involved in the insulin signaling pathway, including AKT (protein kinase B) and IR (insulin receptor). HepG2 cells were cultured overnight and incubated with different concentrations of danthron in FBS-free MEM for $8 \mathrm{~h}$. Insulin was then added $(16.7 \mathrm{nmol} / \mathrm{L})$ for another $5 \mathrm{~min}$, and the cells were harvested for Western blot assay. As shown in Figure 5E, HepG2 cells responded to the insulin stimulus and 


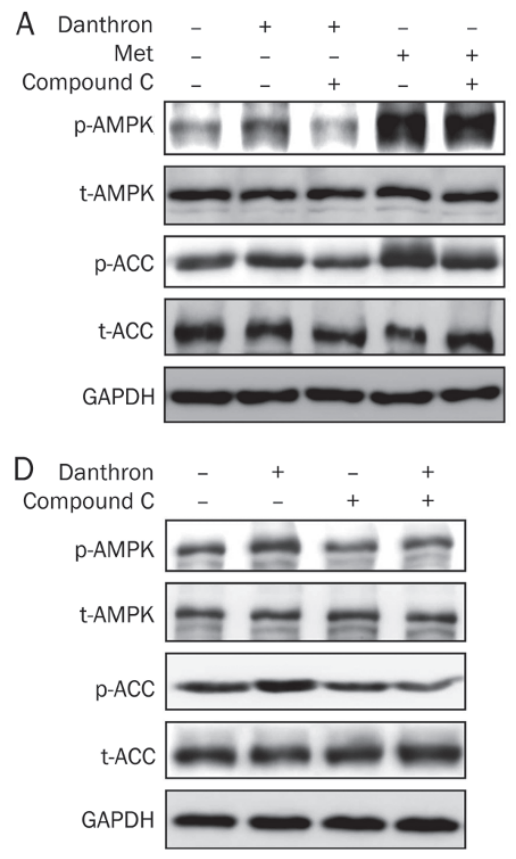

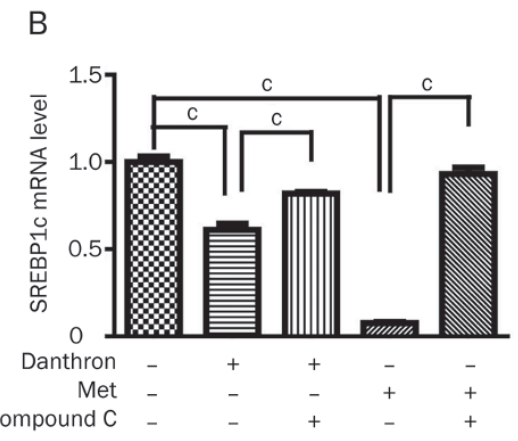

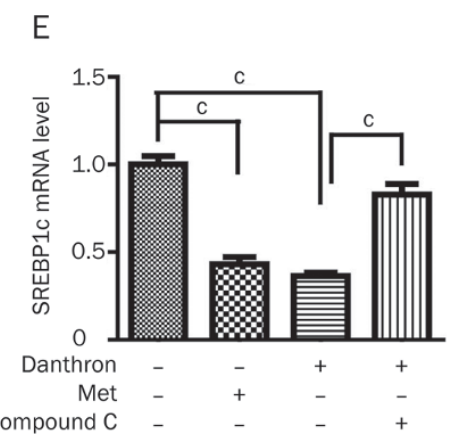

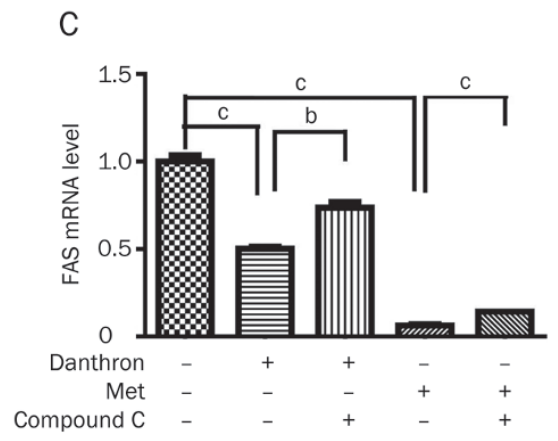

$\mathrm{F}$

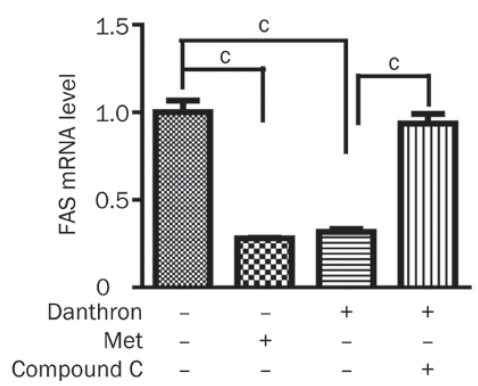

Figure 4. The regulation of the lipid metabolism-related genes by danthron was AMPK-dependent. The phosphorylation of both AMPK and ACC was increased after treatment with danthron and abolished by co-incubation with compound C in both HepG2 (A) and C2C12 (D) cells. Meanwhile, decreases in SREBP1c and FAS mRNA levels induced by danthron were reversed after co-incubation with compound C in HepG2 (B and C) and C2C12 $(\mathrm{E}$ and $\mathrm{F})$ cells. Met $(10 \mathrm{mmol} / \mathrm{L})$ was used as a positive control. The concentrations of both danthron and compound $\mathrm{C}$ were $10 \mu \mathrm{mol} / \mathrm{L}$. Data are presented as the mean \pm SEM of three independent experiments. ${ }^{b} P<0.05,{ }^{C} P<0.01$ vs DMSO or as indicated.

showed significant increases in p-AKT and p-IR. However, no increases in p-AKT or p-IR were observed in the danthrontreated cells; in fact, a downward trend was observed in the concentration of $\mathrm{p}$-IR.

The above-mentioned results suggest that the promotion of glucose uptake by danthron was AMPK-dependent and p-IR/ p-AKT signaling-independent.

\section{Discussion}

Danthron is a natural product extracted from the traditional Chinese medicine rhubarb. In the current work, we determined that this natural product could activate AMPK and ACC activities in both HepG2 and C2C12 cells. Further research has indicated that danthron regulates the gene expressions of lipid synthesis-related SREBP1c and FAS, and cholesterol effluxrelated ABCA1 and ABCG1, reduces intracellular triglyceride and cholesterol levels, and efficiently increases glucose consumption.

Currently, few studies have reported the effects of danthron on glucose and lipid metabolism. It has been reported that danthron could modestly inhibit $\mathrm{Na}^{+}, \mathrm{K}^{+}$-ATPase activity, while it had no effect on glucose transport in the intestine ${ }^{[24]}$. Our previous results showed that danthron as an RXRa antagonist functioned to improve insulin tolerance in diet-induced obese mice $^{[21]}$. Here, we discovered a new pharmacological function of danthron as an activator of AMPK. To identify the potential effects of danthron on AMPK activation and the related regulation of glucose and lipid metabolisms, we detected the levels of AMPK and ACC phosphorylation and their downstream gene expressions in cultured hepatic and skeletal cell lines. As expected, danthron dose-dependently increased the activation of AMPK and ACC (Figures $1 \mathrm{~B}$ and $3 \mathrm{~A})$ and inhibited the downstream SREBP1c and FAS gene expressions. These actions ultimately caused efficient reduction in the intracellular lipid content. The above-mentioned actions of danthron were abolished or partially reversed by AMPK inhibitor compound C. It was suggested that the effect of danthron on lipid metabolism was primarily due to its activation of AMPK. In addition, we preliminarily attempted to evaluate the effects of danthron in diet-induced obese mice, and the results demonstrated modest reduction in serum cholesterol and triglycerides in danthron-treated mice (data not shown).

Notably, an interesting finding in our study was that danthron increased the expressions of ABCA1 and ABCG1. ABCA1 and ABCG1 are known to be involved in cholesterol efflux $^{[25]}$ and are expressed in hepatic and skeletal muscle cells $^{[25-27]}$. Moreover, studies have revealed the regulation of such gene expression by AMPK in endothelial cells ${ }^{[28]}$. Our present results (Figure 2) showed that danthron could increase the transcriptional activities and gene expressions of ABCA1 and ABCG1 dose-dependently in HepG2 and C2C12 cells. This finding explained the possible mechanism for why danthron decreased TC content. Nevertheless, danthron as 
A
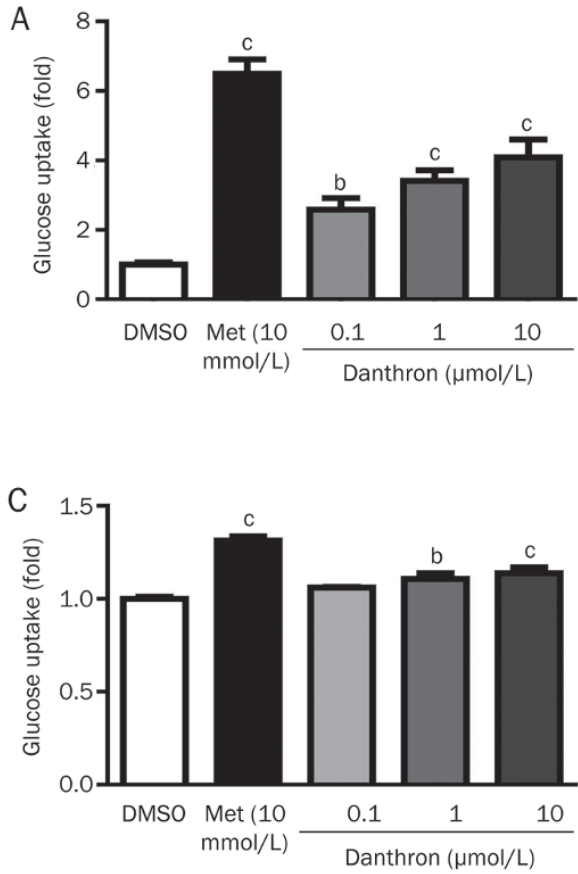

B

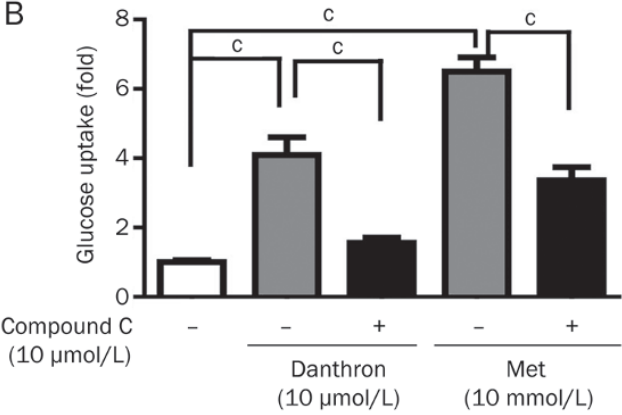

D

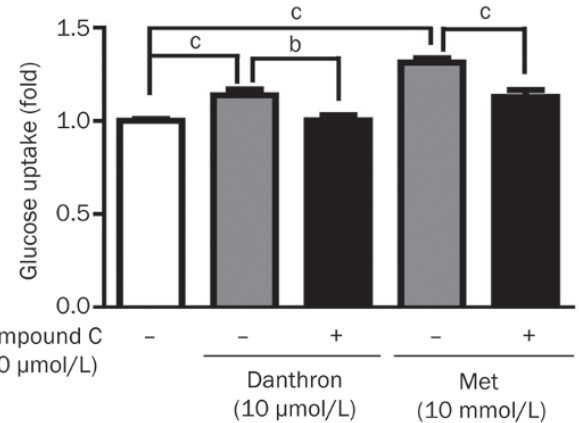

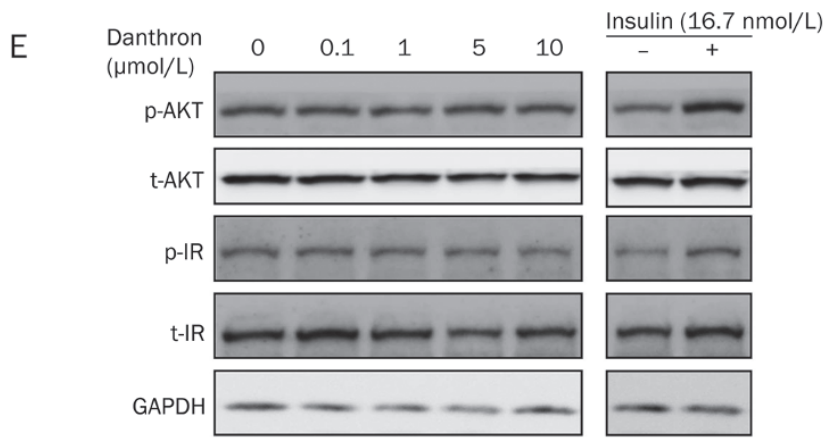

an antagonist of the retinoic $\mathrm{X}$ receptor a/liver $\mathrm{X}$ receptor a (RXRa/LXRa) heterodimer may negatively affect the expressions of $A B C A 1$ and $A B C G 1^{[21,29-31]}$. Thus, the effects of danthron on the ABCA1 and ABCG1 gene expressions seem to be contradictory and complicated. The details regarding the regulation of lipid metabolism by danthron are obscure and require further clarification.

Similarly, in the glucose consumption assay, the potential effects of danthron as the RXRa antagonist on insulin signaling were detected, and no beneficial changes were obtained (Figure 5E). However, the attenuated glucose uptake by compound $\mathrm{C}$ co-incubation with danthron revealed that danthron exerted such effects mainly through the activation of AMPK in the process. Under this condition, the increased Glut4 translocation by AMPK activation promoted the uptake of extracellular glucose in $\mathrm{C} 2 \mathrm{C} 12$ cells $^{[8,32]}$. However, further studies are needed to elucidate whether the AMPK-induced Glut2-dependent glucose uptake existed and contributed to the lowered glucose content in HepG2 cells.

Considering the anti-proliferative and pro-apoptotic prop- erties of danthron, we identified the safe concentration at the beginning of the experiments. Thus, the results we obtained exhibited the real effects of danthron rather than cytotoxic effects. In this study, we discovered a new effect of danthron on glucose and lipid metabolism in both HepG2 and $\mathrm{C} 2 \mathrm{C} 12$ cells through the activation of AMPK. As previously reported, another anthraquinone derivative, emodin, was deemed to activate AMPK by an indirect mechanism similar to berberine ${ }^{[33]}$, but no explicit evidence has been provided. Considering that danthron and emodin have similar effects and chemical structures, we hypothesized that their effects on AMPK may be similar. Thus, further study is needed to identify whether danthron acts as the direct or indirect activator of AMPK, and structural modification may be necessary for drug development in the future. Briefly, our work has determined that danthron is a new, active, natural product that induces the activation of AMPK. Danthron effectively regulated lipid and glucose metabolism. Our findings are expected to prolong the new lipid-lowering function of danthron and to provide a new clue in the search for new anti-diabetic drug discovery. 


\section{Acknowledgements}

This work was supported by the National Science and Technology Major Project (grants 2012ZX09301001-004 and 2012ZX09103101-18), the National Natural Science Foundation of China (grants 81173105 and 91213306), the Foundation of Chinese Academy of Sciences (grant KSCX2-EW-Q-3), the National Marine 863 Programme (grant 2011AA09070102) and the Science Foundation of Shanghai (grant 11XD1406100).

\section{Author contribution}

Prof $\mathrm{Xu}$ SHEN and Prof Li-li CHEN supervised the project; Rong ZHOU, Ling WANG, and Xing XU performed the experiments; Jing CHEN and Li-hong $\mathrm{HU}$ analyzed data; $\mathrm{Xu}$ SHEN, Li-li CHEN, and Rong ZHOU contributed to the writing of the manuscript.

\section{References}

1 DeFronzo RA, Ferrannini E. Insulin resistance. A multifaceted syndrome responsible for NIDDM, obesity, hypertension, dyslipidemia, and atherosclerotic cardiovascular disease. Diabetes Care 1991; 14: 173-94.

2 Grundy SM. Obesity, metabolic syndrome, and cardiovascular disease. J Clin Endocrinol Metab 2004; 89: 2595-600.

3 Kahn BB, Alquier T, Carling D, Hardie DG. AMP-activated protein kinase: ancient energy gauge provides clues to modern understanding of metabolism. Cell Metab 2005; 1: 15-25.

4 Zhang BB, Zhou G, Li C. AMPK: an emerging drug target for diabetes and the metabolic syndrome. Cell Metab 2009; 9: 407-16.

5 Yu LF, Qiu BY, Nan FJ, Li J. AMPK activators as novel therapeutics for type 2 diabetes. Curr Top Med Chem 2010; 10: 397-410.

6 Hawley SA, Davison M, Woods A, Davies SP, Beri RK, Carling D, et al. Characterization of the AMP-activated protein kinase kinase from rat liver and identification of threonine 172 as the major site at which it phosphorylates AMP-activated protein kinase. J Biol Chem 1996; 271: 27879-87.

7 Luo Z, Saha AK, Xiang X, Ruderman NB. AMPK, the metabolic syndrome and cancer. Trends Pharmacol Sci 2005; 26: 69-76.

8 Im SS, Kwon SK, Kim TH, Kim HI, Ahn YH. Regulation of glucose transporter type 4 isoform gene expression in muscle and adipocytes. IUBMB Life 2007; 59: 134-45.

9 Horike N, Sakoda H, Kushiyama A, Ono H, Fujishiro M, Kamata H, et al. AMP-activated protein kinase activation increases phosphorylation of glycogen synthase kinase 3 beta and thereby reduces CAMPresponsive element transcriptional activity and phosphoenolpyruvate carboxykinase $\mathrm{C}$ gene expression in the liver. J Biol Chem 2008; 283 : 33902-10.

10 Cool B, Zinker B, Chiou W, Kifle L, Cao N, Perham M, et al. Identification and characterization of a small molecule AMPK activator that treats key components of type 2 diabetes and the metabolic syndrome. Cell Metab 2006; 3: 403-16.

11 Corton JM, Gillespie JG, Hawley SA, Hardie DG. 5-aminoimidazole-4carboxamide ribonucleoside. A specific method for activating AMPactivated protein kinase in intact cells? FEBS J 1995; 229: 558-65.

12 Walker J, Jijon HB, Diaz H, Salehi P, Churchill T, Madsen KL. 5-aminoimidazole-4-carboxamide riboside (AICAR) enhances GLUT2-dependent jejunal glucose transport: a possible role for AMPK. Biochem J 2005; 385: 485-91.

13 Zang M, Zuccollo A, Hou X, Nagata D, Walsh K, Herscovitz H, et al. AMP-activated protein kinase is required for the lipid-lowering effect of metformin in insulin-resistant human HepG2 cells. J Biol Chem 2004; 279: 47898-905.

14 Lee YS, Kim WS, Kim KH, Yoon MJ, Cho HJ, Shen Y, et al. Berberine, a natural plant product, activates AMP-activated protein kinase with beneficial metabolic effects in diabetic and insulin-resistant states. Diabetes 2006; 55: 2256-64.

15 Lu Y, Yang JH, Li X, Hwangbo K, Hwang SL, Taketomi Y, et al. Emodin, a naturally occurring anthraquinone derivative, suppresses IgEmediated anaphylactic reaction and mast cell activation. Biochem Pharmacol 2011; 82: 1700-8.

16 Niu Y, Li S, Na L, Feng R, Liu L, Li Y, et al. Mangiferin decreases plasma free fatty acids through promoting its catabolism in liver by activation of AMPK. PLoS One 2012; 7: e30782.

17 Liu J, Zhang JF, Lu JZ, Zhang DL, Li K, Su K, et al. Astragalus polysaccharide stimulates glucose uptake in L6 myotubes through AMPK activation and AS160/TBC1D4 phosphorylation. Acta Pharmacol Sin 2013; 34: 137-45.

18 Toyoda K, Nishikawa A, Furukawa F, Kawanishi T, Hayashi Y, Takahashi M. Cell proliferation induced by laxatives and related compounds in the rat intestine. Cancer Lett 1994; 83: 43-9.

19 Lu HF, Lai TY, Hsia TC, Tang YJ, Yang JS, Chiang JH, et al. Danthron induces DNA damage and inhibits DNA repair gene expressions in GBM 8401 human brain glioblastoma multiforms cells. Neurochem Res 2010; 35: 1105-10.

20 Rossi S, Tabolacci C, Lentini A, Provenzano B, Carlomosti F, Frezzotti S, et al. Anthraquinones danthron and quinizarin exert antiproliferative and antimetastatic activity on murine B16-F10 melanoma cells. Anticancer Res 2010; 30: 445-9.

21 Zhang H, Zhou R, Li L, Chen J, Chen L, Li C, et al. Danthron functions as a retinoic $X$ receptor antagonist by stabilizing tetramers of the receptor. J Biol Chem 2011; 286: 1868-75.

22 Mantena SK, Sharma SD, Katiyar SK. Berberine inhibits growth, induces $G_{1}$ arrest and apoptosis in human epidermoid carcinoma A431 cells by regulating Cdki-Cdk-cyclin cascade, disruption of mitochondrial membrane potential and cleavage of caspase 3 and PARP. Carcinogenesis 2006; 27: 2018-27.

23 Huang $\mathrm{H}, \mathrm{Yu}$ Y, Gao Z, Zhang Y, Li C, Xu X, et al. Discovery and optimization of 1,3,4-trisubstituted-pyrazolone derivatives as novel, potent, and nonsteroidal farnesoid $X$ receptor ( $F X R$ ) selective antagonists. J Med Chem 2012; 55: 7037-53.

24 Chignell CF. The effect of phenolphthalein and other purgative drugs on rat intestinal $\left(\mathrm{Na}^{+}+\mathrm{K}^{+}\right)$adenosine triphosphatase. Biochem Pharmacol 1968; 17: 1207-12.

25 Genvigir FD, Rodrigues AC, Cerda A, Arazi SS, Willrich MA, Oliveira R, et al. Effects of lipid-lowering drugs on reverse cholesterol transport gene expressions in peripheral blood mononuclear and HepG2 cells. Pharmacogenomics 2010; 11 : 1235-46.

26 Kase ET, Thoresen GH, Westerlund S, Hojlund K, Rustan AC, Gaster $M$. Liver $X$ receptor antagonist reduces lipid formation and increases glucose metabolism in myotubes from lean, obese and type 2 diabetic individuals. Diabetologia 2007; 50: 2171-80.

27 Oram JF, Vaughan AM. ATP-Binding cassette cholesterol transporters and cardiovascular disease. Circ Res 2006; 99: 1031-43.

28 Li D, Zhang Y, Ma J, Ling W, Xia M. Adenosine monophosphate activated protein kinase regulates ABCG1-mediated oxysterol efflux from endothelial cells and protects against hypercholesterolemiainduced endothelial dysfunction. Arterioscler Thromb Vasc Biol 2010; 30: 1354-62.

29 Uehara Y, Miura S, von Eckardstein A, Abe S, Fujii A, Matsuo Y, et al. Unsaturated fatty acids suppress the expression of the ATP-binding cassette transporter G1 (ABCG1) and ABCA1 genes via an LXR/RXR 
responsive element. Atherosclerosis 2007; 191: 11-21.

30 Field FJ, Born E, Mathur SN. LXR/RXR ligand activation enhances basolateral efflux of beta-sitosterol in CaCo-2 cells. J Lipid Res 2004; 45: 905-13.

31 Murthy S, Born E, Mathur SN, Field FJ. LXR/RXR activation enhances basolateral efflux of cholesterol in CaCo-2 cells. J Lipid Res 2002; 43: 1054-64.
32 Klip A, Schertzer JD, Bilan PJ, Thong F, Antonescu C. Regulation of glucose transporter 4 traffic by energy deprivation from mitochondrial compromise. Acta Physiol (Oxf) 2009; 196: 27-35.

33 Chen Z, Zhang L, Yi J, Yang Z, Zhang Z, Li Z. Promotion of adiponectin multimerization by emodin: a novel AMPK activator with PPARgammaagonist activity. J Cell Biochem 2012; 113: 3547-58. 